\title{
PAPEL DA ESCOLA E DA EDUCAÇÃO FÍSICA NO CONTROLE DA OBESIDADE INFANTIL NA PERCEPÇÃO DE ACADÊMICOS DE EDUCAÇÃO FÍSICA ${ }^{64}$
}

\author{
Jairo Antônio Paixão \\ Universidade Federal de Ouro Preto, Ouro Preto, Minas Gerais, Brasil \\ Maria Teresa Sudário Rocha \\ Universidade Federal de Ouro Preto, Ouro Preto, Minas Gerais, Brasil
}

\begin{abstract}
Resumo
Foi analisado o papel da escola e da Educação Física no controle da obesidade infantil na percepção de acadêmicos de licenciatura em Educação Física. A amostra foi constituída por 100 sujeitos de ambos os sexos que se encontravam no último ano do curso. Foram empregados os questionários Perceptions of Youth Obesity and Physical Education Questionnaire e The Antifat Attitudes Scale. Os acadêmicos reconhecem as consequências da obesidade para a saúde e qualidade de vida e apontam a escola e as aulas de Educação Física como um espaço privilegiado de intervenção para o seu controle. No entanto, os participantes da pesquisa mostraram-se avessos à possibilidade de uma conversão total da Educação Física escolar para essa finalidade.
\end{abstract}

Palavras-chave: Obesidade. Infância. Escola. Educação Física.

\section{Introdução}

Nas últimas décadas, tamanha tem sido a incidência da obesidade entre pessoas de diferentes faixas etárias e classes sociais que muitos estudiosos a têm conceituado como uma epidemia de abrangência sem precedentes no mundo inteiro (GREENLEAF; WEILLER, 2005; EVANS, 2005; COSTA; SOUZA; OLIVEIRA, 2012; GALATTI; SEOANE, 2012). Em alguns estudos, chega a ser definida como doença de origem multifatorial que, por sua vez, estabelece interação entre fatores genéticos, ambientais, socioeconômicos, endócrinos e/ou metabólicos (MARCHI-ALVES et al., 2011).

64 Artigo resultante de pesquisa que teve como órgão financiador o Conselho Nacional de Pesquisa (CNPq) no período de 01 de agosto de 2013 a 31 de julho de 2014 . 
A obesidade tem sido recorrente e crescente na população infantil causando inúmeros problemas de saúde (NASCIMENTO, 2012). Na maioria das vezes, está relacionada ao estilo de vida sedentário, ao fato de a criança permanecer mais tempo em frente à televisão, em participar por um longo período de tempo de jogos virtuais, não brincar na rua por falta de segurança, e ainda, a mudanças nos hábitos alimentares, pois preferem produtos não nutritivos, ricos em gorduras e calorias, que apresentam maior facilidade no seu preparo (ANDERSON; BUTCHER, 2006). Cabe lembrar que, nesta fase da vida, menos de $10 \%$ dos casos de sobrepeso e obesidade decorrem de fatores endócrinos (COSTA; SOUZA; OLIVEIRA, 2012; MARCHI-ALVES et al., 2011; NASCIMENTO, 2012). Nessa perspectiva, estudos longitudinais (MUST, 1996; ROSSNER, 1998) envolvendo sujeitos obesos mostraram que a obesidade na infância e na adolescência tende a continuar na fase adulta, se não for convenientemente controlada, podendo ser a causa do aumento no índice de morbimortalidade e diminuição da expectativa de vida.

A escola como uma instituição educadora formal - que a primeira vista poderia ser vislumbrada como um espaço em potencial para se abordar a obesidade - acaba por reproduzir e, em alguns casos, acirrar conflitos e atitudes preconceituosas e discriminatórias para com a criança obesa a exemplo do que comumente ocorre em diferentes espaços sociais (COSTA; SOUZA; OLIVEIRA, 2012).

Mais especificamente, as aulas de Educação Física se configuram como um ambiente em que, muitas vezes, a exclusão dos alunos com sobrepeso e obesos se assevera a partir da ocorrência de inúmeras práticas preconceituosas e discriminatórias como, por exemplo, em momentos em que ocorrem mudanças de roupas, o uso de uniformes inadequados ao obeso e as dificuldades em completar as atividades físicas, em comparação com seus colegas que se encontram na condição física eutrófica (GALATTI; SEOANE, 2012).

Em contrapartida, a Educação Física cuja maioria dos conteúdos apresenta o movimento como eixo comum pode trabalhar o desenvolvimento das habilidades motoras, a aptidão física e possibilitar a conscientização dos alunos sobre a importância de um estilo de vida ativo. Destacando também que uma alimentação saudável representa valiosa ação educativa para o controle do sobrepeso e obesidade, implicando assim diretamente na melhoria dos padrões de saúde e qualidade de vida do adulto em potencial.

Para a efetivação das referidas ações no ambiente escolar, os professores de Educação Física se revestem de conhecimentos na área da saúde e de forma mais profícua, no que se refere à condução de programas voltados à aptidão física, além do trabalho de processos de conscientização acerca de hábitos saudáveis nos alunos (GREENLEAF; WEILLER, 2005).

A partir dessas considerações, o objetivo do presente estudo foi analisou as percepções de acadêmicos do curso de licenciatura em Educação Física com relação à obesidade infantil e o papel da escola e da Educação Física como formas de controle.

\section{Métodos}

O presente trabalho caracterizou-se como um estudo descritivo-exploratório (THOMAS; NELSON; SILVERMAN, 2012). O grupo amostral foi constituído de 100 acadêmicos do curso de licenciatura em Educação Física de ambos os sexos (49 homens e 51 mulheres), buscando assegurar uma dimensão de modo a obter uma amostra representativa para o presente estudo, o número de sujeitos foi determinado pelo pesquisador no viés de amostragem por conveniência. 
O grupo amostral derivou-se das seguintes instituições de ensino localizadas no estado de Minas Gerais: Universidade Federal de Ouro Preto, Universidade Federal de Juiz de Fora, Faculdade de Minas - Campus Muriaé, Faculdade vértice. A escolha das referidas instituições se deu por conveniência. O critério de inclusão foi o tempo em que esses sujeitos se encontravam matriculados no curso, sendo selecionados aqueles que cursavam o último ano (dois últimos períodos do curso). A opção por este critério diz respeito à vivência e relações estabelecidas pelos acadêmicos com os saberes pedagógicos, científicos e experienciais que alicerçam a formação e, por conseguinte, a prática docente (TARDIF, 2011).

Para a coleta de dados, foram utilizadas as versões traduzidas para o português dos seguintes instrumentos: Perceptions of Youth Obesity and Physical Education Questionnaire (PRICE; DESMOND; RUPPERT, 1990) para avaliar a percepção do papel da Educação Física na diminuição do sobrepeso e obesidade de crianças. Esse questionário contém 12 itens que se referem a fatores que podem contribuir para a obesidade e outros 30 itens sobre o problema da obesidade na infância e o papel da escola e da Educação Física em lidar com os problemas da obesidade. Na operacionalização das variáveis contidas nos itens, o questionário estrutura-se a partir da escala Likert de 5 pontos, em que o informante poderia marcar uma alternativa $(1=$ discordo plenamente; 2=discordo; $3=$ =indiferente; 4=concordo; 5=concordo plenamente) em ordem de importância, de acordo com a percepção dos temas relacionados à obesidade na infância. Para fins de apresentação dos resultados, as categorias 1 e 2 foram consideradas negativas, a 3 neutra e as categorias 4 e 5 positivas. Foi utilizado também The Antifat Attitudes Scale (MORRISON; O'CONNOR, 1999), um questionário que contém cinco itens designados para medir atitudes negativas com pessoas com sobrepeso. Este instrumento estrutura-se a partir da escala Likert de 5 pontos, em que o informante poderia marcar uma alternativa ( $1=$ discordo plenamente; $2=$ discordo; $3=$ indiferente; $4=$ concordo; $5=$ concordo plenamente) com a que mais se identificasse com relação às pessoas obesas.

A tradução para o português dos instrumentos utilizados para a coleta de dados foi realizada pelo pesquisador, que buscou assegurar a veracidade da versão original, visando, sobretudo, evitar e/ou minimizar risco na forma de interpretação por parte dos sujeitos participantes do estudo.

Os referidos instrumentos foram inseridos em formulários no Google Drive (https://drive.google.com/), estruturados a partir de uma mensagem inicial com informações básicas sobre a pesquisa, links de acesso aos instrumentos de coleta de dados e ao Termo de Consentimento Livre e Esclarecido (TCLE) na íntegra, bem como o endereço eletrônico do pesquisador reponsável, caso se fizesse necessário o estabelecimento de contato por parte dos participantes. Os contatos dos sujeitos foram cedidos pelos dirigentes dos cursos de Licenciatura em Educação Física das instituições de ensino superior que se propuseram a participar. A coleta de dados se deu no período de dezembro de 2013 a março de 2014. Foram encaminhados e-mails para um total de 178 sujeitos. Dentro do tempo estimado para essa fase do estudo, obteve-se o retorno por um total de 100 sujeitos, que foram considerados como grupo amostral.

O estudo foi aprovado pelo Comitê de Ética em Pesquisa com seres humanos da Universidade Federal de Ouro Preto, registro número 291.921 e CAAE 16837613.4.0000.5150. 


\section{RESULTADOS}

Os resultados apresentados na Tabela 1 versam sobre a percepção dos entrevistados sobre a obesidade infantil, implicações com a saúde e prevalência de possíveis resultados obtidos por meio de programas voltados ao emagrecimento.

TABELA 1 - Percepção sobre a obesidade infantil

\begin{tabular}{|c|c|c|c|c|c|}
\hline \multirow{2}{*}{ VARIÁVEIS CONSIDERADAS } & \multicolumn{5}{|c|}{ ESCALA DE LIKERT (\%) } \\
\hline & 1 & 2 & 3 & 4 & 5 \\
\hline $\begin{array}{l}\text { A obesidade infantil está se convertendo em um tema cada vez mais } \\
\text { relevante. }\end{array}$ & 2 & 3 & 0 & 37 & 58 \\
\hline $\begin{array}{l}\text { O fracasso na hora de perder peso, após participar de um programa } \\
\text { destinado a esse fim, reforça as crenças do jovem de que existe uma } \\
\text { pequena oportunidade de conseguir essa perda. }\end{array}$ & 3 & 21 & 11 & 52 & 13 \\
\hline Ter um peso normal é muito importante para a saúde das crianças. & 1 & 3 & 7 & 42 & 47 \\
\hline $\begin{array}{l}\text { As pessoas obesas são sexualmente menos atraentes que as pessoas } \\
\text { magras. }\end{array}$ & 12 & 22 & 18 & 32 & 16 \\
\hline A obesidade infantil é uma causa significativa de rejeição entre iguais. & 0 & 15 & 11 & 59 & 15 \\
\hline Muitas crianças superarão sua obesidade. & 6 & 30 & 26 & 31 & 7 \\
\hline Nunca sairia com uma pessoa obesa. & 41 & 28 & 21 & 8 & 2 \\
\hline $\begin{array}{l}\text { Com a ajuda apropriada, muitas crianças obesas são capazes de perder uma } \\
\text { considerável quantidade de peso. }\end{array}$ & 0 & 0 & 1 & 45 & 54 \\
\hline No geral, as pessoas obesas são mais preguiçosas que as pessoas magras. & 8 & 22 & 19 & 39 & 12 \\
\hline $\begin{array}{l}\text { Com a ajuda apropriada, muitas crianças obesas que perderam peso são } \\
\text { capazes de manter essa perda. }\end{array}$ & 1 & 2 & 2 & 56 & 39 \\
\hline
\end{tabular}

1=Discordo plenamente; 2=Discordo; 3=Indiferente; 4=Concordo; 5=Concordo Plenamente

Esses resultados indicaram a existência de consenso entre os entrevistados (95\%) sobre a relevância que se acentua sobre a obesidade entre a população infantil nos últimos tempos. Somado a esse fator, tem-se a percepção da dificuldade e mesmo da grande incidência de fracassos nas tentativas de perder peso por meio de programas destinados a essa finalidade. $74 \%$ dos sujeitos investigados concordam que a rejeição na população infantil apresenta-se como uma das implicações da obesidade nessa fase da vida. No que se refere à saúde, $89 \%$ dos respondentes relacionaram o peso ideal com a manutenção da saúde na infância. Ao serem questionados sobre a prevalência dos resultados obtidos em programas voltados à perda de peso, $95 \%$ dos participantes reconhecem que crianças que contam com apoio e ajuda apropriada serão capazes, não somente de obter perdas mais significativas de peso, bem como de manter essas perdas ao longo da vida. 
A Tabela 2 apresenta dados relacionados à visão dos alunos sobre o papel da escola no controle da obesidade infantil. Foram consideradas as seguintes variáveis: o tratamento da problemática nas instituições e medidas a serem tomadas para a melhoria no quadro atual.

TABELA 2 - O Papel da escola no trato da obesidade infantil

\begin{tabular}{|c|c|c|c|c|c|}
\hline \multirow{2}{*}{ VARIÁVEIS CONSIDERADAS } & \multicolumn{5}{|c|}{ ESCALA DE LIKERT (\%) } \\
\hline & 1 & 2 & 3 & 4 & 5 \\
\hline As escolas deveriam dispor de cardápios com baixas calorias. & 0 & 5 & 9 & 43 & 43 \\
\hline $\begin{array}{l}\text { Os pais não deveriam converter a escola em um lugar para o tratamento da } \\
\text { obesidade infantil. }\end{array}$ & 4 & 11 & 12 & 40 & 33 \\
\hline $\begin{array}{l}\text { A bulimia e a anorexia nervosa são problemas mais sérios dentro do contexto escolar } \\
\text { que a obesidade. }\end{array}$ & 7 & 30 & 25 & 24 & 14 \\
\hline $\begin{array}{l}\text { As escolas não se envolvem o suficiente para ajudar a combater o problema da } \\
\text { obesidade. }\end{array}$ & 2 & 7 & 8 & 58 & 25 \\
\hline $\begin{array}{l}\text { Todas as escolas deveriam dispor de aulas de E F especificamente pensadas para as } \\
\text { crianças obesas. }\end{array}$ & 16 & 33 & 15 & 25 & 11 \\
\hline $\begin{array}{l}\text { As escolas deveriam dispor de programas de controle de peso destinados a tratar a } \\
\text { obesidade infantil. }\end{array}$ & 1 & 6 & 12 & 60 & 21 \\
\hline $\begin{array}{l}\text { Deveriam eliminar-se das cantinas das escolas os alimentos ricos em gordura, balas } \\
\text { e doces. }\end{array}$ & 6 & 10 & 15 & 40 & 29 \\
\hline $\begin{array}{l}\text { O professor regente deveria fazer, na escola, o controle e tratamento da obesidade } \\
\text { nas crianças. }\end{array}$ & 9 & 29 & 21 & 33 & 8 \\
\hline $\begin{array}{l}\text { As escolas deveriam dispor de matérias curriculares que contenham conteúdos sobre } \\
\text { nutrição e controle de peso. }\end{array}$ & 1 & 9 & 10 & 50 & 30 \\
\hline
\end{tabular}

1=Discordo plenamente; 2=Discordo; 3=Indiferente; 4=Concordo; 5=Concordo Plenamente

Os resultados demonstram que, no tocante aos alimentos distribuídos nas escolas, $86 \%$ dos sujeitos investigados acreditam que os cardápios deveriam conter apenas alimentos de baixas calorias. Por outro lado, com relação à postura a ser adotada pela escola para amenizar o quadro atual de obesidade infantil, $73 \%$ dos participantes não concordam que a escola deva ser convertida em um ambiente exclusivo para se tratar a obesidade infantil. Foi informado ainda por $80 \%$ dos sujeitos entrevistados que as escolas não têm se envolvido o suficiente com a temática. Ainda, com relação ao papel da escola ante à obesidade infantil, a atuação do professor regente como ator coadjuvante no tratamento da obesidade surge como alternativa por $41 \%$ dos participantes da pesquisa.

Especificando o objetivo final da pesquisa, a Tabela 3 apresenta a forma como os sujeitos percebem a Educação Física no controle direto à obesidade e no fomento de um estilo de vida 
saudável dos alunos. Nesse sentido, foram abordadas as variáveis: a duração das aulas, a função do professor da disciplina com relação ao problema, a programação de atividades na escola e a formação inicial no curso de licenciatura em Educação Física para lidar com as questões relacionadas à obesidade de crianças.

TABELA 3 - O papel da Educação Física escolar no trato da obesidade infantil

\begin{tabular}{|c|c|c|c|c|c|}
\hline \multirow{2}{*}{ VARIÁVEIS CONSIDERADAS } & \multicolumn{5}{|c|}{ ESCALA DE LIKERT (\%) } \\
\hline & 1 & 2 & 3 & 4 & 5 \\
\hline $\begin{array}{l}\text { Não se dispõe do tempo suficiente nas aulas de E F para ajudar os jovens a melhorarem } \\
\text { sua forma física. }\end{array}$ & 7 & 13 & 12 & 39 & 29 \\
\hline $\begin{array}{l}\text { Aconselhar as crianças e seus pais sobre os programas de exercícios para perder peso é } \\
\text { uma atividade muito gratificante. }\end{array}$ & 0 & 2 & 6 & 52 & 40 \\
\hline $\begin{array}{l}\text { A formação recebida não me preparou de forma adequada para elaborar um programa de } \\
\text { atividades para ajudar as crianças a reduzir seu peso. }\end{array}$ & 14 & 35 & 17 & 24 & 10 \\
\hline $\begin{array}{l}\text { O professor de EF deveria fazer, na escola, o controle e tratamento da obesidade nas } \\
\text { crianças. }\end{array}$ & 6 & 25 & 17 & 38 & 14 \\
\hline $\begin{array}{l}\text { Acredito que sou competente na hora de prescrever um programa de exercícios destinado } \\
\text { à perda de peso para alunos obesos. }\end{array}$ & 3 & 16 & 19 & 50 & 12 \\
\hline $\begin{array}{l}\text { Conceber programas de atividades para perder peso e aconselhar tanto as crianças quanto } \\
\text { seus pais sobre tais aspectos é uma tarefa muito difícil. }\end{array}$ & 3 & 22 & 7 & 49 & 19 \\
\hline $\begin{array}{l}\text { Acredito que os professores de EF deveriam servir de modelo, sendo exemplo e mantendo } \\
\text { um peso normal. }\end{array}$ & 5 & 17 & 16 & 44 & 18 \\
\hline $\begin{array}{l}\text { Os alunos deveriam receber aulas de EF centradas no cuidado com a forma física ao longo } \\
\text { de todo o ciclo de vida. }\end{array}$ & 8 & 18 & 5 & 32 & 37 \\
\hline $\begin{array}{l}\text { Muitas das aulas de EF não são elaboradas para proporcionar hábitos de vida e padrões de } \\
\text { exercício que ajudem a controlar o peso. }\end{array}$ & 1 & 11 & 9 & 46 & 33 \\
\hline
\end{tabular}

Do total de sujeitos investigados, $67 \%$ acreditam que as aulas de Educação Física deveriam ser elaboradas para ajudar as crianças a melhorar a forma física ao longo da vida, no entanto, reconhece que a carga horária semanal das aulas é insuficiente para que ocorram alterações corporais significativas nas crianças. Apesar disso, esse mesmo percentual afirma que o professor da disciplina deve fazer o controle da obesidade na escola e servir de modelo para os alunos, mantendo o peso dentro dos padrões considerados normais. No que se refere à formação profissional obtida até o último ano do curso de licenciatura, obteve-se um resultado que merece atenção, $49 \%$ afirmaram que o curso os tinha preparado para elaborar programas de atividades para reduzir o excesso de peso em crianças, enquanto $34 \%$ negaram ter recebido essa preparação no curso até aquele momento de formação. 


\section{Discussão}

Os resultados obtidos revelaram que, em concordância com outros estudos (GREENLEAF; WEILLER, 2005; COSTA; SOUZA; OLIVEIRA, 2012; GALATTI; SEOANE, 2012), os acadêmicos do curso de Licenciatura em Educação Física percebem a obesidade como um tema emergente na sociedade atual e ressaltam a importância da adequação da composição corporal ao percentil para a faixa etária tanto na manutenção da saúde como na prevenção de uma série de doenças relacionadas.

A obesidade foi apontada pelos sujeitos desta pesquisa como causa de rejeição entre iguais, corroborando com estudos que avaliaram essa condição, o que acarreta atitudes preconceituosas desde a fase que compreende a infância (GREENLEAF; WEILLER, 2005; COSTA; SOUZA; OLIVEIRA, 2012; STRAUSS; POLLACK, 2003). Esse tipo de rejeição relaciona-se à forma de perceber a pessoa obesa como menos atraente, mais preguiçosa e desmazelada consigo mesma se comparada à pessoa que se encontra com o peso corporal adequado com sua faixa etária e altura. Essa percepção encontra-se impregnada por valores vigentes em uma sociedade que parece prevalecer um padrão de beleza corporal (COSTA; SOUZA; OLIVEIRA, 2012).

De uma maneira geral, os resultados que tratam diretamente sobre a percepção dos acadêmicos sobre a pessoa obesa não revelaram atitudes negativas com relação ao sujeito com a condição de obesidade. Esses resultados apresentam-se favoráveis no que se referem à formação de futuros professores de Educação Física que poderão atuar diretamente com programas voltados à prevenção e tratamento da obesidade infantil na ambiência da Educação Básica.

Quanto às possibilidades de superação do quadro de obesidade entre crianças, percebeu-se, nos resultados desta pesquisa, que entre aqueles que creem em tal possibilidade de mudança, a necessidade de um acompanhamento apropriado. Nessa perspectiva, estudos relacionados ao tema evidenciam que a perda de peso corporal torna-se possível mediante mudanças no estilo de vida, alimentação e exercícios físicos orientados, atuando na regulação do balanço energético (GALATTI; SEOANE, 2012; FERNANDES; PENHA; BRAGA, 2012).

No que se refere ao ambiente escolar, os entrevistados acreditam ser importante eliminar das cantinas alimentos ricos em gordura, balas e doces. Tal crença encontra-se em consonância com o Programa Nacional de Alimentar Escolar - PNAE (2013), que define o oferecimento pela escola em torno de $20 \%$ da ingestão necessária tanto de calorias quanto de nutrientes aos alunos. Essa percepção corrobora com estudos que apontam uma mudança de padrão alimentar nos últimos anos (KAC; SICHIERI; GIGANTE, 2008). Nesse processo de mudanças, é preciso considerar ainda fatores internos e externos que determinam a obesidade, como valores sociais e culturais, influência da família, necessidades e características psicológicas (MELLO; LUFT; MEYER, 2004).

Ainda que a escola possa contribuir como um espaço para instalação e efetivação de programas e políticas públicas para o tratamento da obesidade infantil, os acadêmicos do curso de Licenciatura em Educação Física contestam a total conversão da escola para essa finalidade (GALATTI; SEOANE, 2012). Um estudo semelhante foi realizado com professores de Educação Física que atuavam na Educação Básica nos Estados Unidos e os resultados contradizem a posição dos sujeitos da presente pesquisa ao perceber a escola como um local ideal para se tratar o problema (GREENLEAF; WEILLER, 2005). Em contrapartida, nota-se concordância de opinião entre os sujeitos dos referidos estudos ao afirmarem que as instituições de ensino não se envolvem o suficiente para o combate ao problema e, por sua vez, deveriam dispor de programas de controle do 
peso corporal, bem como a inclusão do tema no currículo escolar. Sobre esse posicionamento dos sujeitos, ainda que seja importante esse tipo de preocupação na escola, torna-se salutar um esforço coletivo que busque envolver a família, a criança e a própria sociedade, haja vista que a obesidade relaciona-se a uma infinidade de fatores como os culturais, econômicos, emocionais e comportamentais (EVANS, 2005; LUIZ et al., 2005).

Considerando que no ambiente da escola, dentre as disciplinas e conteúdos que compreendem o currículo dos segmentos que Educação Básica, a Educação Física configura-se como área do saber em que o professor detém conhecimentos relacionados à saúde e qualidade de vida mediatizados por um estilo de vida ativo e pelas práticas corporais. Por sua vez, um profissional habilitado a procedimentos como orientar e sensibilizar crianças sobre hábitos saudáveis (FERNANDES; PENHA; BRAGA, 2012). Nessa perspectiva, ao se questionar os sujeitos se as escolas deveriam dispor das aulas de Educação Física para atendimento de crianças obesas, a maioria discordou dessa possibilidade. Esses resultados podem estar relacionados ao fato da percepção desses acadêmicos do curso de licenciatura em Educação Física sobre os demais objetivos e finalidades atribuídos à Educação Física como prática de intervenção na escola de Educação Básica. Esses objetivos incluem para além dos conhecimentos sobre o corpo e suas práticas, aquelas relacionadas às diferentes manifestações culturais produzidas historicamente pelo homem, dentre outras que também se fazem importantes ser tratadas nas aulas de Educação Física escolar (SOARES et al., 1992).

Foi apontada ainda, pela maioria dos entrevistados, a insuficiência de tempo destinado às aulas de Educação Física nas escolas. No Brasil, na maioria das vezes, a presença ou não da disciplina no currículo da Educação Infantil e anos iniciais, bem como a carga horária semanal acaba ficando a cargo da escola. Não há uma regulamentação que normatiza essa situação. Assim, é muito comum, nos referidos segmentos da Educação Básica, a permuta da aula de Educação Física por aulas com professores especialistas como línguas, artes e outras (MINAS GERAIS, 2013). Fundamentando-se através de experiências pregressas no meio escolar como alunos da Educação Básica, ao comentar sobre as aulas, os entrevistados ressaltaram que, na maioria das vezes, elas não são planejadas de maneira a proporcionar hábitos de uma vida saudável e padrões de exercício para controlar o peso, mas acreditam que isso deveria ser feito, sendo elaboradas aulas centradas no cuidado com a forma física ao longo do ciclo de vida. De acordo com Greenleaf e Weiller (2005), os professores de Educação Física devem encorajar a atividade física, sendo essas realizadas dentro da escola e levadas, pelos alunos, para outros contextos, caracterizando assim a educação para o movimento, atitude defendida pelos Parâmetros Curriculares Nacionais (BRASIL, 1997; BRASIL, 2000). Têm-se ainda diferentes metodologias de ensino da Educação Física escolar e uma delas é a da Saúde Renovada (GUEDES; GUEDES, 1996; NAHAS, 1997), que ressalta a importância da Educação Física voltada à saúde, relacionando a importância dos conhecimentos práticos e teóricos de atividade física, aptidão física e saúde, para dar ao aluno entendimento dos benefícios da atividade física e de utilizá-los por toda vida.

Ao se considerar a formação inicial em licenciatura, suas finalidades e a escola como campo de intervenção docente, questionamentos foram realizados no sentido de abstrair se os acadêmicos se sentem preparados e competentes para elaborar um programa de atividades para a redução de peso de escolares. Os acadêmicos que se encontram no final do processo de formação de um curso de licenciatura acreditam estar preparados para trabalhar aspectos relacionados à obesidade na escola. Esse fato que leva a pensar que os acadêmicos buscam informações e saberes específicos a 
partir de outras fontes que extrapolam os cursos de Licenciatura em Educação Física sobre o tema obesidade, como participação em congressos, seminários e mesmo a realização de disciplinas do bacharelado na condição de disciplinas eletivas. Trata-se de uma situação que desponta com potencial de ser analisada.

\section{Considerações Finais}

Diante das constatações obtidas nesta investigação e considerando as suas limitações metodológicas, é possível afirmar que a análise realizada a partir da visão de acadêmicos do curso de Licenciatura em Educação Física sobre a obesidade infantil e juvenil e o papel da escola e da Educação Física obteve resultados relevantes na proposta desse estudo.

Os entrevistados veem a importância da problemática bem como a necessidade de intervenção para prevenir e tratar a obesidade em crianças e jovens e reconhecem a rejeição sofrida por esse grupo em consequência de sua condição. Constatou-se também a importância das instituições formadoras nesse processo, com o implemento de projetos e programas a fim de contribuir para abrandar o quadro atual de sobrepeso e obesidade no Brasil.

De acordo com os participantes, a escola configura-se como espaço que detém potencial para a realização de intervenções, programas e matérias curriculares voltados para o controle da obesidade infantil e juvenil. Asseveram, no entanto, que a referida instituição não deve ser convertida em um lugar onde essa meta seja o foco principal.

Verificou-se ainda que os entrevistados acreditam que, dentro da escola, o profissional de Educação Física é aquele que se encontra em condições para lidar com diferentes compleições físicas, podendo desenvolver em suas aulas procedimentos que possam contribuir para que crianças e jovens mantenham a forma. Nesse sentido, a educação para e pelo movimento, se constitui caminho necessário na efetivação de um estilo de vida saudável e bons hábitos alimentares. Concluem que a aula de Educação Física é o principal contexto para retratar a obesidade, apesar da restrição da carga horária dessa disciplina na escola. Para tanto, os entrevistados se sentem preparados para desenvolver programas para esses fins.

$\mathrm{Na}$ realização do presente estudo, algumas limitações se fizeram presentes como a dificuldade em obter retorno dos dados, via e-mail, por parte dos sujeitos participantes que, por sua vez, implicou diretamente no prazo definido para conclusão da etapa de coleta de dados e ainda na dificuldade de se trabalhar com grupo amostral maior. Assim, sugerem-se novas investigações sobre o tema, incluindo observação de aulas, análise aprofundada de planejamento e de matrizes curriculares dos cursos de formação e, ainda, possibilidades de acesso e articulação dos saberes relacionados à obesidade e seus procedimentos por professores licenciados em Educação Física.

\section{Agradecimentos/Financiamentos}

Agradecimentos ao Conselho Nacional de Pesquisa (CNPq). 


\title{
THE ROLE OF SCHOOL AND PHYSICAL EDUCATION IN PREVENTING CHILDHOOD OBESITY IN THE PERCEPTION OF PHYSICAL EDUCATION STUDENTS
}

\begin{abstract}
The study analyzed the role of school and Physical Education in preventing childhood obesity, according to the perception of Physical Education students. The sample consisted of 100 subjects of both sexes who were attending the last year of the under-graduation course of Physical Education. Researchers used the Perceptions of Youth Obesity and Physical Education Questionnaire and The Antifat Attitudes Scale. The students recognize the implications of obesity for health and the quality of life, and highlighted the importance of the school and Physical Education classes for the prevention of childhood obesity. However, the subjects were against the possibility of full conversion of the school for this purpose.
\end{abstract}

Keywords: Obesity. Childhood. School. Physical Education.

\section{PAPEL DE LA ESCUELA E DE LA EDUCACIÓN FÍSICA EN EL CONTROL DE LA OBESIDAD INFANTIL EN LA PERCEPCIÓN DE ACADÉMICOS DE EDUCACIÓN FÍSICA}

\section{Resumen}

Fue analizado el papel de la escuela y de la Educación Física en el control de la obesidad infantil en la percepción de los académicos de licenciatura en Educación Física. La muestra fue constituida por 100 personas de ambos los sexos que se encontraban en el último año del curso. Fueron aplicados los cuestionarios Perceptions of Youth Obesity and Physical Education Questionnaire y The Antifat Attitudes Scale. Los académicos reconocen las consecuencias de la obesidad para la salud y cualidad de vida y apuntan la escuela y las clases de Educación Física como un espacio privilegiado de intervención para su control. Sin embargo, los participantes de la investigación se mostraron contrarios a la posibilidad de una conversión total de la Educación Física escolar para esa finalidad.

Palabras-clave: Obesidad. Infancia. Escuela. Educación Física.

\section{Referências}

ANDERSON, P. M.; BUTCHER, K. E. Childhood obesity: trends and potential causes. Future Child. v. 16, n.1, p.19-45, 2006. Disponível em: <http://futureofchildren.org/futureofchildren/publications/docs/16-01-02.pdf >. Acesso em: 04 de nov. 2013.

BRASIL. Secretaria de Educação Fundamental. Parâmetros curriculares nacionais: Educação física /Secretaria de Educação Fundamental - Brasília: MEC/SEF, 1997.

Ministério da Educação. Secretaria de Educação Média e Tecnológica. Parâmetros Curriculares Nacionais (Ensino Médio). Brasília: MEC, 2000. 
COSTA, M. A. P.; SOUZA, M. A.; OLIVEIRA, V. M. Obesidade infantil e bullying: a ótica dos professores. Educação e Pesquisa, v. 38, n. 3, p. 653-665, 2012. Disponível em:< http://www.educacaoepesquisa.fe.usp.br/>. Acesso em10 de abril de 2014.

EVANS, W. D.; et al. Public Perceptions of Childhood Obesity. American Journal of Preventive Medicine, v. 28, n. 1, p.26-32, 2005.

FERNANDES, M. M.; PENHA, D. S. G.; BRAGA, F. A. Obesidade infantil em crianças da rede pública de ensino: prevalência e consequências para flexibilidade, força explosiva e velocidade. Revista da Educação Física /UEM, v. 23, n.4, p. 629-634, 2012. Disponível em:< http://www.periodicos.uem.br/ojs/index.php/RevEducFis > Acesso em 15 de fevereiro de 2014.

GALATTI, L. R.; SEOANE, M. A.; PAES, R. R. Pedagogia do Esporte e Obesidade: perspectivas para um estilo de vida saudável a partir da adequada iniciação esportiva na infância. Pensar a Prática, Goiânia, v. 15, n.2, p. 452-467, 2012.

GREENLEAF, C., WEILLER, K. Perceptions of youth obesity among physical educators Social Psychology of Education, v. 8, n. 4, p.407- 423, 2005.

GUEDES, D.P; GUEDES, J. E. R. P Controle do Peso Corporal: Composição Corporal Atividade Física e Nutrição. Londrina: Midiograf, 1996.

KAC, G.; SICHIERI, R.; GIGANTE, D. P. Epidemiologia nutricional. Rio de Janeiro: Editora Fiocruz, 2008.

LUIZ, A. M. A. G. et al. Depressão, ansiedade, competência social e problemas comportamentais em crianças obesas. Estudos de Psicologia, Natal, v. 10, n. 3, p.371-375, set./dez. 2005.

MARCHI-ALVES, L. M. et al. Obesidade infantil ontem e hoje: importância da avaliação antropométrica pelo enfermeiro. Escola Anna Nery, Rio de Janeiro,v.15, n. 2, p. 238-244, abr./jun.2011.

MELLO, E. D.; LUFT, V. C.; MEYER, F. Obesidade infantil: como podemos ser eficazes?. Jornal de Pediatria, Porto Alegre, v. 80, n. 3, p.173-182, maio/jun.2004.

MINAS GERAIS. Secretaria de Educação Estadual. Resolução 2.253/13 artigo 4º. SEE, 2013.

MORRISON, T. G.; O'CONNOR, W. E. Psychometric properties of a scale measuring negative attitudes toward overweight individuals. The Journal of Social Psychology, v. 139, n.4, p.436$445,1999$.

MUST, A. Morbidity and mortality associated with elevated body weight in children and adolescents. Am J Clin Nutr, v.63 (suppl): p.445-7, 1996. Disponível em: http://ajcn.nutrition.org/content/63/3/445S.full.pdf+html Acesso em: 30/08/2014. 
NAHAS, M. V. Educação Física no Ensino Médio: educação para um estilo de vida ativo no terceiro milênio. In: Seminário de Educação Física Escolar/Escola de Educação Física e Esportes, USP. Anais... p.17-20, 1997.

NASCIMENTO, V. G. et al. Prevalence of overweight preschool children in public day care centers: a cross-sectional study. São Paulo Medical Journal, v. 130, n. 4, p. 225-229, 2012.

PRICE, J. H.; DESMOND, S. M.; RUPPERT, E. S. Elementary physical education teachers' perceptions of childhood obesity. Health Education, v.21, n.8, p.26-32, 1990.

PROGRAMA NACIONAL DE ALIMENTAR ESCOLAR (PNAE). Resolução/CD/FNDE no 26, de 17 de junho de 2013. Disponível $\quad$ em:< http://www.fnde.gov.br/fnde/legislacao/resolucoes/item/4620-resolu\%C3\%A7\%C3\%A3o-cd-fnden\%C2\%BA-26,-de-17-de-junho-de-2013>. Acesso em: 07-maio de 2014.

ROSSNER, S. Childhood obesity and adulthood consequenses. Acta Pediatr, v.87, p.1-5., 1998. Disponível em: http://www.ncbi.nlm.nih.gov/pubmed/9510438 Acesso em: 26/08/2014

SOARES, C. et al. Metodologia do ensino de educação física. São Paulo: Cortez, 1992.

STRAUSS, R. S.; POLLACK, H. A. Social marginalization of overweight children. Archives of Pediatric and Adolescent Medicine, v.157, n.8, p.746-752, 2003.

TARDIF, M. Saberes docentes e formação profissional. 14. ed. Petrópolis: Vozes, 2011.

THOMAS, J. R.; NELSON, J. K; SILVERMAN, S. J. Métodos de pesquisa em atividade física. 6. ed. Porto Alegre: Artmed, 2012.

Recebido em:12/12/2014

Revisado em: 16/04/2015

Aprovado em: 17/04/2015

Endereço para correspondência:

Jairo Antônio Paixão

jairopaixao2004@yahoo.com.br

Universidade Federal de Ouro Preto, Reitoria.

Morro do Cruzeiro s/n

Centro

35400-000 - Ouro Preto, MG - Brasil 